\title{
Roughness exponents to calculate multi-affine fractal exponents
}

\author{
A. Castro e Silva, J.G. Moreira \\ Departamento de Fisica, Instituto de Ciências Exatas, Universidade Federal de Minas Gerais, C.P. 702, \\ 30161-970, Belo Horizonte, MG, Brazil
}

Received 2 August 1996

\begin{abstract}
We propose a new method to determine the multi-affine fractal exponents based on a generalization of the roughness concept. For synthetic multi-affine fractal profiles with exactly known exponents, we show that the results obtained in this method are more accurate than the results obtained with the height-height correlation function method. Also in the present method, scaling is observed using profiles with a number of points more than one order of magnitude smaller.
\end{abstract}

PACS: $02.70 ; 06.30 \mathrm{C} ; 68.20$

The concept of self-affine fractals has opened a wide area of research because it represents a very powerful tool in the investigation of many complex systems found in nature. These structures have been found in physical systems like surface growing [1,2], fractures [3] and biological systems like DNA patterns [4] and human heartbeat [5]. The most important characteristics of a self-affine fractal is the roughness exponent $H$ which is a measure of variation of its thickness with the scaling factor. Several methods have been used to calculate the thickness and the roughness exponent, namely, the variation method [6,7], the roughness methods $[7,8]$, and the height-height correlation function [9].

Another type of fractals that appear in nature are the multifractals. These geometric structures have an infinite number of singularities and therefore need an infinite set of exponents to properly describe these singularities [10]. The extension of the multifractal ideas to self-affine fractals give rise to the multi-affine fractal concept $[9,11]$. In this case, the profiles cannot be described by just one roughness exponent $H$, but by an infinite number of scaling exponents related to the $q$ th order of the moments of the

${ }^{*}$ Corresponding author. E-mail: jmoreira@fisica.ufmg.br. 
distribution considered, which obey the multiscaling power law $W_{q}(\varepsilon) \sim \varepsilon^{q H_{q}}$, where $\varepsilon$ is a small scaling factor. This concept of multi-affinity is important in the study of interfaces, such as, models of kinetic surface roughening [12,13]. In this work we use a generalization of the roughness concept in order to describe these multi-affine fractals.

Before defining multi-affine fractal exponents we are going to discuss how the roughness exponent is calculated in self-affine fractals. Let us consider a discrete profile $f\left(x_{i}\right)$ with $L$ points, where $x_{i}$ is the coordinate of the $i$ th point, and $f\left(x_{i}\right)$ is a single value function. The roughness $W(\varepsilon)$ of these profiles $f\left(x_{i}\right)$ is the average thickness over a neighborhood of size $\varepsilon$, defined as

$$
W(\varepsilon)=\frac{1}{L} \sum_{i=1}^{L} w\left(x_{i}, \varepsilon\right)
$$

where $w\left(x_{i}, \varepsilon\right)$ is an appropriate measure of the local thickness, or roughness, of the profile $f\left(x_{i}\right)$ in the interval $\left[x_{i}-\varepsilon, x_{i}+\varepsilon\right]$. For example, the local roughness $w\left(x_{i}, \varepsilon\right)$ can be evaluated by $(\mathrm{A})$ the difference between the maximum and the minimum height [6,7]; (B) the dispersion around the local average height [7]; (C) the height-height correlation function [9]; and (D) the dispersion around the best linear least-square fitting [8]. The roughness $W(\varepsilon)$ exhibits a single scaling power law given by

$$
W(\varepsilon) \sim \varepsilon^{H}
$$

It is possible to relate this method with a cover of the profile $f\left(x_{i}\right)[6,8]$. Of these methods, the method $(D)$ is the one that provides the finest cover for the profile $f\left(x_{i}\right)$, therefore, giving a better estimate for the roughness exponent $H$ [8]. In this method, the local roughness $w\left(x_{i}, \varepsilon\right)$ is defined as

$$
w^{2}\left(x_{i}, \varepsilon\right)=\frac{1}{(2 \varepsilon+1)} \sum_{y=x_{i}-\varepsilon}^{x_{i}+\varepsilon}[f(y)-(a y+b)]^{2},
$$

where $a$ and $b$ are coefficients to be determined by a least-square fitting.

In the case of multi-affine fractal profiles, the set of exponents $H_{q}$ are usually evaluated using a $q$ th order height-height correlation function [9]

$$
C_{q}(\varepsilon)=\frac{1}{L} \sum_{i=1}^{L}\left|f\left(x_{i}\right)-f\left(x_{i}+\varepsilon\right)\right|^{q},
$$

which behaves as

$$
C_{q}(\varepsilon) \sim \varepsilon^{q H_{q}}
$$

for small $\varepsilon$. It is important to note that $C_{q}(\varepsilon)$ can also be seen as a cover of the profile $f\left(x_{i}\right)$ as discussed above, but now one has a different cover for each moment of the distribution. We can expect that the imperfections of these covers increase when we take higher moments since we are amplifying the errors by a power of $q$. 

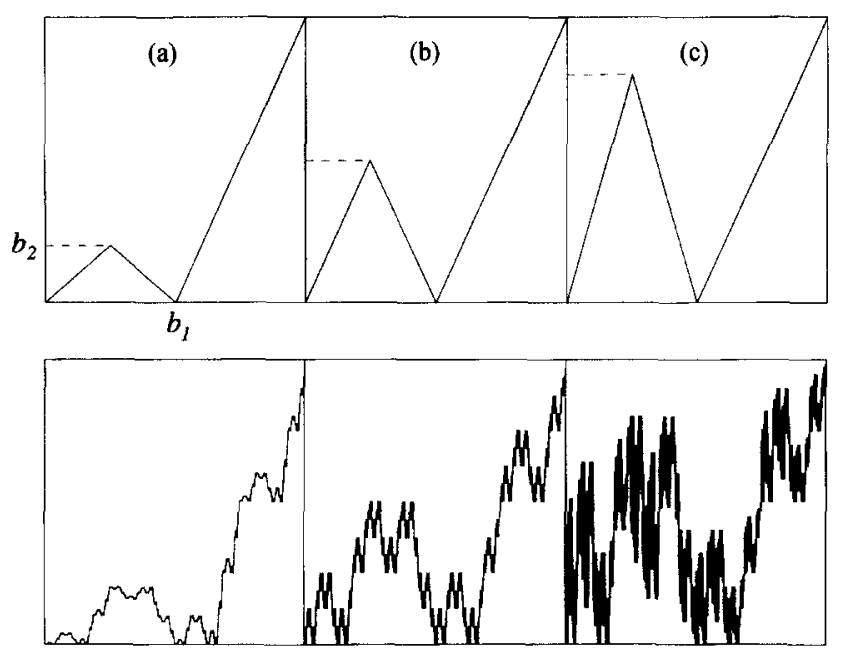

Fig. 1. Synthetic multi-affine profiles with $b_{1}=0.5$ and (a) $b_{2}=0.2$, (b) $b_{2}=0.5$ and (c) $b_{2}=0.8$. At the top of the figure the three seeds and, at the bottom, profiles are presented in the seventh stage $\left(4^{7}+1=16385\right.$ points $)$.

It is possible to generalize the average thickness (1) in a self-affine profile to a multi-affine profile by defining the $q$ th order multi-roughness function as

$$
W_{q}(\varepsilon)=\frac{1}{L} \sum_{i=1}^{L} w_{q}\left(x_{i}, \varepsilon\right),
$$

where the local multi-roughness $w_{q}\left(x_{i}, \varepsilon\right)$ is a generalization of Eq. (3), namely

$$
w_{q}^{2}\left(x_{i}, \varepsilon\right)=\frac{1}{(2 \varepsilon+1)} \sum_{y=x_{i}-\varepsilon}^{x_{i}+\varepsilon}|f(y)-(a y+b)|^{2 q} .
$$

We expect to find for the $W_{q}(\varepsilon)$ the scaling behavior

$$
W_{q}(\varepsilon) \sim \varepsilon^{q H_{q}} .
$$

We propose the definitions (6) and (7) because, in the case of self-affine profiles, it gives a better cover than the one represented by (4), specially for extreme values of $H, H \sim 0.1$ and $H \sim 0.9$ [8]. Moreover, the use of the dispersion around the best linear fitting is very useful when we deal with fractals which have sharp drifts, as in the examples of Fig. 1. These examples are a generalization of a recursively self-affine fractal with $H=0.5$ that generates multi-affine profiles with exponents given exactly by [9]

$$
H_{q}=\frac{\ln \left[\left(b_{1}^{q}+b_{2}^{q}\right) / 2\right]}{q \ln \left(\frac{1}{4}\right)},
$$




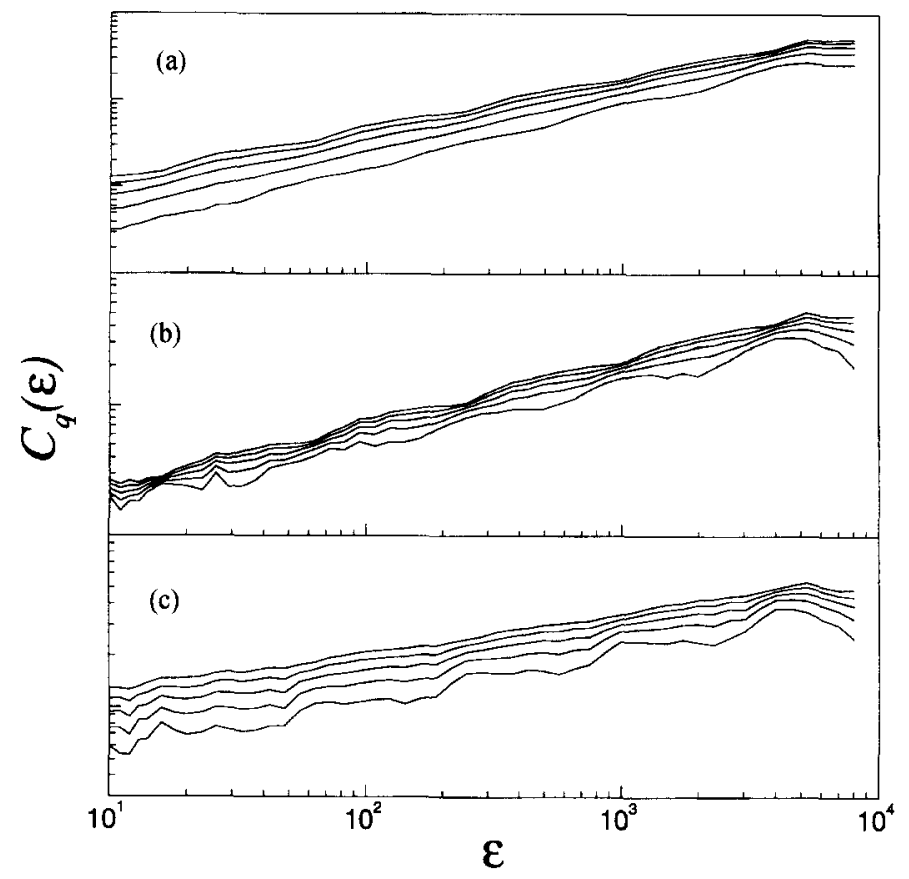

Fig. 2. The $\log -\log$ plot of the height-height correlation function $C_{q}(\varepsilon)$ versus the scale $\varepsilon$ for the first five moments of the three profiles of Fig. 1: (a) $b_{2}=0.2$, (b) $b_{2}=0.5$ and (c) $b_{2}=0.8$ with $b_{1}=0.5$ in the three plots. The order of the moments increases from the bottom to top in each plot.

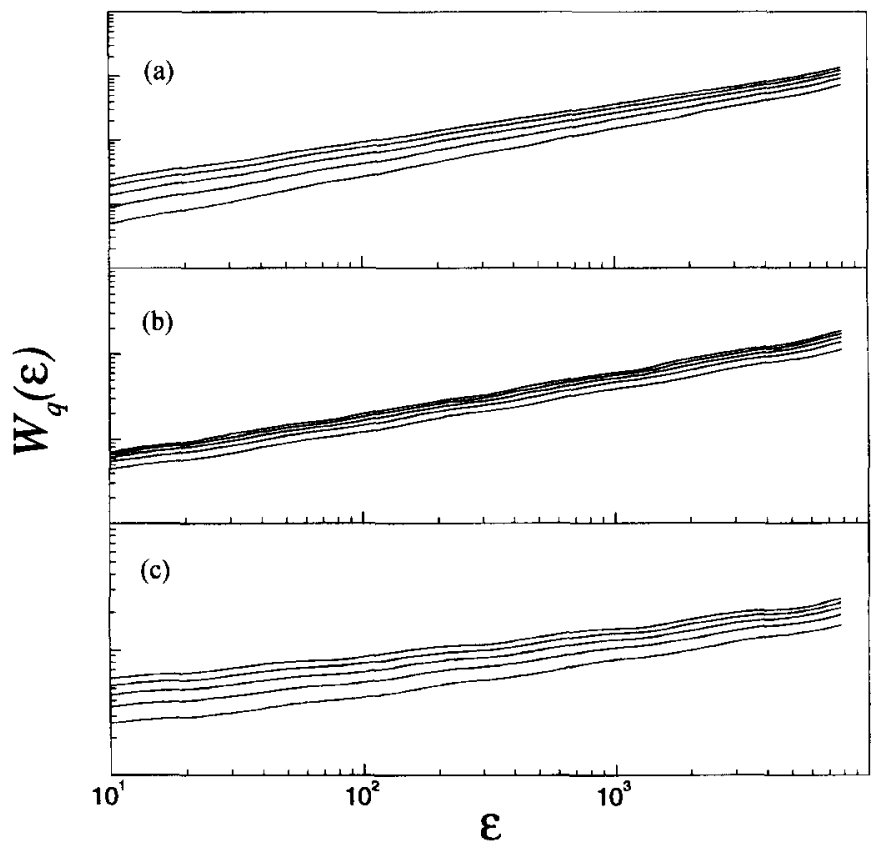

Fig. 3. Same as Fig. 2 for the roughness $W_{q}(\varepsilon)$. 
Table 1

Comparison of the theoretical $H_{t}$ values of the multi-affine exponents with the values obtained by the height-height correlation function method $H_{h}$ and roughness method $H_{W}$ for the three profiles in Fig. 1 and the respective correlation coefficients $r_{h}$ and $r_{W}$

\begin{tabular}{|c|c|c|c|c|c|}
\hline$q$ & $H_{t}$ & $H_{h}$ & $r_{h}$ & $H_{W}$ & $r_{W}$ \\
\hline \multicolumn{6}{|c|}{$b_{2}=0.2$} \\
\hline 1 & 0.757 & 0.712 & 0.9983 & 0.740 & 0.9999 \\
\hline 2 & 0.696 & 0.664 & 0.9992 & 0.683 & 0.9999 \\
\hline 3 & 0.652 & 0.627 & 0.9992 & 0.640 & 0.9999 \\
\hline 4 & 0.620 & 0.600 & 0.9991 & 0.610 & 0.9999 \\
\hline 5 & 0.598 & 0.582 & 0.9989 & 0.590 & 0.9999 \\
\hline \multicolumn{6}{|c|}{$b_{2}=0.5$} \\
\hline 1 & 0.500 & 0.453 & 0.9895 & 0.486 & 0.9995 \\
\hline 2 & 0.500 & 0.467 & 0.9960 & 0.482 & 0.9994 \\
\hline 3 & 0.500 & 0.473 & 0.9975 & 0.482 & 0.9994 \\
\hline 4 & 0.500 & 0.476 & 0.9978 & 0.482 & 0.9994 \\
\hline 5 & 0.500 & 0.478 & 0.9977 & 0.482 & 0.9995 \\
\hline \multicolumn{6}{|c|}{$b_{2}=0.8$} \\
\hline 1 & 0.311 & 0.278 & 0.9825 & 0.297 & 0.9974 \\
\hline 2 & 0.292 & 0.264 & 0.9900 & 0.276 & 0.9970 \\
\hline 3 & 0.275 & 0.249 & 0.9937 & 0.258 & 0.9970 \\
\hline 4 & 0.260 & 0.235 & 0.9957 & 0.243 & 0.9971 \\
\hline 5 & 0.248 & 0.224 & 0.9967 & 0.231 & 0.9971 \\
\hline
\end{tabular}

where $b_{1}$ and $b_{2}$ are defined in Fig. 1. In order to appreciate the power of the method presented here we apply it to these profiles and compare it with the height-height correlation function method.

Fig. 2 shows the graph of the height-height correlation function $C_{q}(\varepsilon)$, defined by Eq. (4), versus the scaling factor $\varepsilon$ for the first five moments of the profiles of Fig. 1. All calculations were done with a pre-fractal in a seventh stage of iteration $(16,385$ points). In this stage the correlation function does not show the expected scaling behavior and it is necessary to go to the nineth stage (262 145 points) in order to obtain reasonable scaling behavior [9].

Fig. 3 shows the graph of the multi-roughness $W_{q}(\varepsilon)$, defined by Eqs. (6) and (7), as a function of the scaling factor $\varepsilon$ for the same profiles also in the seventh stage. Note that, with this method, we obtain much better linear fittings, indicating that the scaling is observed at earlier stages. We calculate the slope $H_{q}$ and the correlation coefficient $r$ ( $r=1$ corresponds to perfect fitting) for the three profiles and first five moments using both methods. Table 1 shows these results and the theoretical values of $H_{q}$ determined by Eq. (9). We note that the multi-roughness method presented here gives an estimate of the exponents $H_{q}$ better than the correlation method.

In order to compare the convergence of the two methods, we calculate the exponent of the first moment $H_{1}$ for some pre-fractals at different stages of the iterative process. 


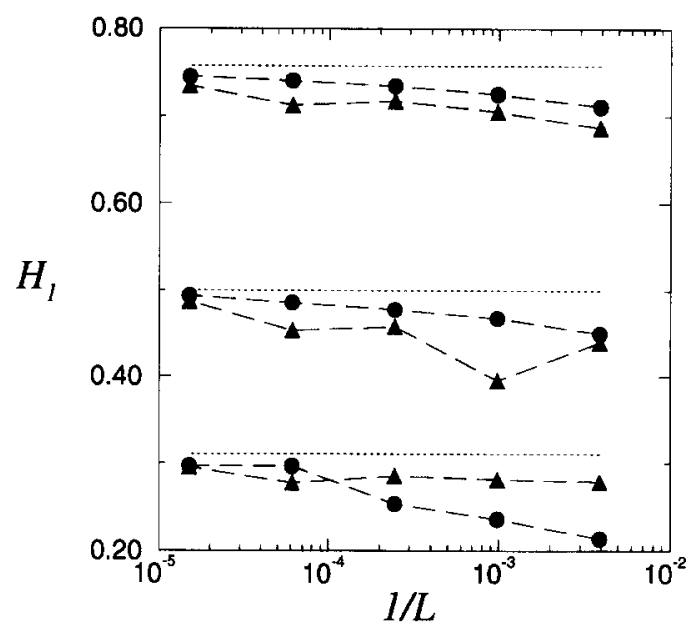

Fig. 4. The linear-log plot of the exponent of the first moment $H_{1}$ for some pre-fractals at different stages as a function of $1 / L$. The triangles indicate the correlation method and the circles the roughness method. The dotted line is the exact value and the dashed lines are guides to the eyes.

A pre-fractal in the $k$-stage has $L=4^{k}+1$ points and Fig. 4 shows the values of $H_{1}$ obtained with the two methods in function of $1 / L$ for $4 \leqslant k \leqslant 8$. We note that the roughness method presents a consistent convergent behavior towards the exact value. For the correlation method, however, we observe that it seems to approach to the exact value, but in an irregular fashion.

In conclusion, we introduce a method to determine the multi-affine fractal exponents $H_{q}$ which is based on a generalization of the roughness exponent (Eqs. (6) and (7)). We also compare the height-height correlation function method with the present one and we show that the latter provides better results and also scaling is observed at much earlier stages. Furthermore, the values obtained with the present method converge to the exact value in a more consistent manner.

\section{Acknowledgements}

The authors wish to thank Sylvie Oliffson Kamphorst for useful discussions. We thank also Osiel de Alcântara Bonfim for suggestions and helpful criticism of the manuscript. This research was supported in part by Conselho Nacional de Desenvolvimento Científico e Tecnológico $(\mathrm{CNPq})$ and in part by Fundação de Amparo à Pesquisa do Estado de Minas Gerais (Fapemig), Brazilian agencies.

\section{References}

[1] T. Vicsek, Fractal Growth Phenomena (World Scientific, Singapore, 1992).

[2] A.-L. Barabási and H.E. Stanley, Fractal Concepts in Surface Growth (Cambridge, 1995).

[3] B.B. Mandelbrot, D.E. Passoja and A.J. Paullay, Nature 308 (1984) 721. 
[4] C.-K. Peng, S.V. Buldyrev, A.L. Goldberger, S. Havlin, F. Sciortino, M. Simons and H.E. Stanley, Nature 356 (1992) 168; H.E. Stanley, S.V. Buldyrev, A.L. Goldberger, Z.D. Goldberger, S. Havlin, R.N. Mantegna, S.M. Ossadnik, C.-K. Peng and M. Simons, Physica A 205 (1994) 214.

[5] C.-K. Peng, J. Mietus, J.M. Hausdorff, S. Havlin, H.E. Stanley and A.L. Goldberger, Phys. Rev. Lett. 70 (1993) 1343.

[6] B. Dubuc, J.F. Quiniou, C. Roques-Carmes, C. Tricot and S.W. Zucker, Phys. Rev. A 39 (1989) 1500.

[7] J. Schmittbuhl, J.P. Vilotte and S. Roux, Phys. Rev. E 51 (1995) 131.

[8] J.G. Moreira, J. Kamphorst Leal da Silva and S. Oliffson Kamphorst, J. Phys. A 27 (1994) 8079.

[9] A.-L. Barabási and T. Vicsek, Phys. Rev. A 44 (1991) 2730.

[10] T.C. Halsey, M.H. Jensen, L.P. Kadanoff, I. Procaccia and B.I. Shaiman, Phys. Rev. A 33 (1986) 1141.

[11] A.-L. Barabási, P. Szépfalusy and T. Vicsek, Physica A 178 (1991) 17.

[12] A.-L. Barabási, R. Bourbonnais, M. Jensen, J. Kertész, T. Vicsek and Y.-C. Zhang, Phys. Rev. A 45 (1992) R6951.

[13] S. Das Sarma, C.J. Lanczycki, R. Kotliar and S.V. Ghaisas, Phys. Rev. E 53 (1996) 359. 